\title{
ASYMPTOTIC EQUIVALENCE OF A LINEAR AND NONLINEAR SYSTEM WITH IMPULSE EFFECT
}

\author{
by P. S. SIMEONOV and D. D. BAINOV
}

(Received 17th July 1986)

\section{Introduction}

The present paper deals with the problem of asymptotic equivalence of the system with impulse effect

$$
\frac{d x}{d t}=A x+g(t), \quad t \neq t_{k} ;\left.\quad \Delta x\right|_{t=t_{k}}=B x\left(t_{k}\right)
$$

and

$$
\frac{d y}{d t}=A y+g(t)+f(t, y), \quad t \neq t_{k} ;\left.\quad \Delta y\right|_{t=t_{k}}=B y\left(t_{k}\right)+b_{k}\left(y\left(t_{k}\right)\right),
$$

where $x, y: I \rightarrow R^{n} ; g: I \rightarrow R^{n} ; f: I \times R^{n} \rightarrow R^{n} ; b_{k}: R^{n} \rightarrow R^{n} ; I=[0, \infty) ; R^{n}$ is the $n$-dimensional Euclidean space with a norm $|\cdot| ; A$ and $B$ are constant matrices; the moments $\left\{t_{k}\right\}$ constitute an increasing sequence $0<t_{1}<\cdots<t_{k}<\cdots, \lim _{k \rightarrow \infty} t_{k}=\infty$.

The systems with impulse effect of type (1) are characterized by the fact that at the moments $\left\{t_{k}\right\}$ under the action of instant effect (impulse), the mapping point $(t, x)$ jumps from the position $\left(t_{k}, x\left(t_{k}\right)\right)$ into the position $\left(t_{k}, x\left(t_{k}\right)+\Delta x\left(t_{k}\right)\right)$. It is also supposed that at the moments of impulse effect $\left\{t_{k}\right\}$ the solutions of systems (1) and (2) are left continuous, i.e. $x\left(t_{k}-0\right)=x\left(t_{k}\right),\left.\Delta x\right|_{t=t_{k}}=x\left(t_{k}+0\right)-x\left(t_{k}\right)$.

We shall make use of the following definition for asymptotic equivalence.

Definition 1. The systems (1) and (2) are said to be asymptotically equivalent if there is a one-to-one correspondence between their solutions such that

$$
\lim _{t \rightarrow \infty}|x(t)-y(t)|=0
$$

for the corresponding solutions $x(t)$ and $y(t)$.

The main theorem of this paper is an analogue of the theorem of Brauer [1] for asymptotic equivalence of systems without impulse effect.

\section{Preliminary remarks}

Further on the following notation is used: $i(t, s)$-the number of the points $t_{k}$ inside the interval $(t, s) ;\|A\|=\sup _{|x|=1}|A x|$-the norm of the matrix $A=\left(a_{i j}\right)_{1}^{n} ; E$-the unit $n \times n$ 
matrix; $0_{m}$-the zero $m \times m$ matrix; $\operatorname{diag}\left(A_{1}, A_{2}\right)$-the quasidiagonal $n \times n$ matrix with blocks $A_{1}$ and $A_{2}$.

In the proof of our main result we shall use the following lemma.

Lemma 1 [2]. Let the following conditions be fulfilled:

1. The function $u: I \rightarrow I$ is piecewise continuous on I being left continuous at the points of discontinuity $\left\{t_{k}\right\}$, and $0<t_{1}<\cdots<t_{k}<\cdots, \lim _{k \rightarrow \infty} t_{k}=\infty$.

2. The function $\lambda: I \rightarrow R$ is continuous on $I$ and the numbers $d_{k}, k=0,1, \ldots$, are nonnegative.

3. For $t \in I$ the inequality

$$
u(t) \leqq d_{0}+\int_{0}^{t} \lambda(s) u(s) d s+\sum_{0<t_{k}<t} d_{k} u\left(t_{k}\right)
$$

holds.

Then for $t \in I$ the following inequality holds

$$
u(t) \leqq d_{0} \prod_{t_{0}<t_{k}<t}\left(1+d_{k}\right) \exp \left(\int_{0}^{t} \lambda(s) d s\right) .
$$

Denote by (A) the following set of conditions:

A1. All solutions of system (1) are bounded on $I$.

A2. Constants $Q>0$ and $p>0$ exist such that

$$
\left|i\left(t_{0}, t\right)-p\left(t-t_{0}\right)\right| \leqq Q, \quad \text { for } \quad 0 \leqq t_{0} \leqq t<\infty .
$$

A3. The function $g: I \rightarrow R^{n}$ is continuous on $I$.

A4. $\operatorname{det}(E+B) \neq 0$ and the matrices $A$ and $B$ commute.

A5. The inverse functions $h_{k}^{-1}$ of the functions $h_{k}: R^{n} \rightarrow R^{n}, h_{k}(y)=y+B y+\mathscr{C}_{k}(y)$, $k=1,2, \ldots$, exist.

.A6. The functions $f: I \times R^{n} \rightarrow R^{n}$ and $b_{k}: R^{n} \rightarrow R^{n}, k=1,2, \ldots$ are continuous on their domains and a non-negative continuous function $\lambda: I \rightarrow I$ and non-negative constants $\beta_{k}$, $k=1,2, \ldots$ exist, such that

$$
\begin{aligned}
&|f(t, y)| \leqq \lambda(t)|y|, \quad \text { for } t \in I \text { and } y \in R^{n}, \\
&|f(t, y)-f(t, z)| \leqq \lambda(t)|y-z| \text { for } t \in I \text { and } y, z \in R^{n}, \\
&\left|b_{k}(y)\right| \leqq \beta_{k}|y|, \text { for } y \in R^{n}, \quad k=1,2, \ldots, \\
&\left|b_{k}(y)-b_{k}(z)\right| \leqq \beta_{k}|y-z|, \text { for } y, z \in R^{n}, \quad k=1,2, \ldots, \\
& \int_{0}^{\infty} \lambda(s) d s+\sum_{k=1}^{\infty} \beta_{k} \leqq L<\infty
\end{aligned}
$$




\section{Main results}

Theorem 1. Let the conditions (A) be fulfilled. Then the systems (1) and (2) with impulse effect are asymptotically equivalent.

Proof. Let $x(t)=x\left(t ; t_{0}, x_{0}\right)$ be a solution of (1) such that $x\left(t_{0}+0\right)=x_{0}$, and $y(t)=$ $y\left(t ; t_{0}, x_{0}\right)-$ a solution of (2) such that $y\left(t_{0}+0\right)=x_{0}$.

We are going to show that for $t_{0}$ sufficiently large, there exists a one-to-one correspondence between the initial states $x_{0}$ and $y_{0}$ which in turn generates a one-toone correspondence between the solutions $x(t)$ and $y(t)$ of systems (1) and (2). Further on we shall demonstrate that for each two corresponding solutions the relation (3) holds.

The general solution $X$ of the linear non-homogeneous system (1) has the form

$$
X=\eta+Z
$$

where $\eta$ is any solution of (1) and $Z$ is the general solution of the linear homogeneous system

$$
\frac{d z}{d t}=A z, \quad t \neq t_{k} ;\left.\quad \Delta z\right|_{t=t_{k}}=B z\left(t_{k}\right) .
$$

It follows from A1 and (9) that all solutions of (10) are bounded. Since the matrices $A$ and $B$ commute, then according to [2] the solution $z(t)=z\left(t ; t_{0}, x_{0}\right)$ of $(10)$ has the form

$$
z(t)=(E+B)^{i\left(t_{0}, t\right)} e^{A\left(t-t_{0}\right)}, \text { for } t>t_{0},
$$

or

$$
z(t)=(E+B)^{i\left(t_{0}, t\right)-p\left(t-t_{0}\right)} e^{\Lambda\left(t-t_{0}\right)} z_{0}, \text { for } t>t_{0}
$$

where $\Lambda=A+p \ln (E+B)$.

Bearing in mind the boundedness of the solution $z(t)$ and condition $A 2$, it follows from (11) that the matrix $e^{\Lambda\left(t-t_{0}\right)}$ is bounded for $0 \leqq t_{0} \leqq t<\infty$. Hence the matrix $\Lambda$ has the structure $\Lambda=S^{-1} \operatorname{diag}\left(\Lambda_{-}, \Lambda_{0}\right) S$, where $\Lambda_{-}$is a $q \times q$ Jordan matrix whose eigenvalues $\lambda_{i}$ have negative real parts $\operatorname{Re} \lambda_{i}<-\alpha<0, i=1, \ldots, q ; \Lambda_{0}$ is a $r \times r$ Jordan matrix whose eigenvalues $\mu_{i}$ have zero real parts and simple elementary divisors, $\operatorname{Re} \mu_{j}=0, j=1, \ldots, r ; q+r=n ; \operatorname{det} S \neq 0$.

Introduce the matrix functions

$$
\begin{gathered}
G(t, s)= \begin{cases}(E+B)^{i(s, t)-p(t-s)} S^{-1} \operatorname{diag}\left(e^{\Lambda-(t-s)}, e^{\Lambda 0(t-s)}\right) S, & t>s ; \\
(E+B)^{-i(t, s)+p(s-t)} S^{-1} \operatorname{diag}\left(e^{\Lambda-(t-s)}, e^{\Lambda_{0}(t-s)}\right) S, & t \leqq s ;\end{cases} \\
G_{-}(t, s)= \begin{cases}(E+B)^{i(s, t)-p(t-s)} S^{-1} \operatorname{diag}\left(e^{\Lambda-(t-s)}, O_{r}\right) S, & t>s ; \\
(E+B)^{-i(t, s)+p(s-t)} S^{-1} \operatorname{diag}\left(e^{\Lambda-(t-s)}, O_{r}\right) S, & t \leqq s ;\end{cases} \\
G_{0}(t, s)= \begin{cases}(E+B)^{i(s, t)-p(t-s)} S^{-1} \operatorname{diag}\left(O_{q}, e^{\Lambda_{0}(t-s)}\right) S, & t>s ; \\
\left.(E+B)^{-i(t, s)+p(s-t)} S^{-1} \operatorname{diag} O_{q}, e^{\Lambda_{0}(t-s)}\right) S, & t \leqq s\end{cases}
\end{gathered}
$$




\section{P. S. SIMEONOV AND D. D. BAINOV}

An immediate verification shows that

$$
\begin{gathered}
G(t, s)=G_{-}(t, s)+G_{0}(t, s), \\
G(t, t)=E, \text { for } t \in I, \\
G\left(t_{k}+0, t_{k}\right)=E, \\
G\left(t_{k}+0, s\right)-G\left(t_{k}, s\right)=B G\left(t_{k}, s\right), \text { for } s<t_{k}, \\
\frac{\partial U}{\partial t}=A U \text { for } t \neq t_{k},
\end{gathered}
$$

where $U$ is any of the matrices $G, G_{-}$or $G_{0}$.

Then the solution $x(t)$ of $(1)$ is of the form

$$
x(t)=G\left(t, t_{0}\right) x_{0}+\int_{i_{0}}^{t} G(t, s) g(\mathrm{~s}) d s
$$

Using (15)-(18) we see that the solution $y(t)$ of (2) satisfies, for $t>t_{0}$, the equation

$$
y(t)=G\left(t, t_{0}\right) y_{0}+\int_{t_{0}}^{t} G(t, s) g(s) d s+\int_{t_{0}}^{t} G(t, s) f(s, y(s)) d s+\sum_{t_{0}<t_{k}<t} G\left(t, t_{k}\right) b_{k}\left(y\left(t_{k}\right)\right)
$$

Bearing in mind the structure of the matrix $\Lambda, A 2,(12)$ and (13), the following estimates for the matrices $G_{-}, G_{0}$ and $G$ can be obtained

$$
\begin{aligned}
& \left\|G_{-}(t, s)\right\| \leqq a \exp (-\alpha(t-s)), \quad \text { for } \quad 0 \leqq s \leqq t<\infty, \\
& \left\|G_{0}(t, s)\right\| \leqq a, \quad \text { for } t \in I, s \in I, \\
& \|G(t, s)\| \leqq a, \quad \text { for } \quad 0 \leqq s \leqq t<\infty,
\end{aligned}
$$

where the constant $a>0$ does not depend on $s$ and $t$.

The inequalities (4), (6), (21) and (23) together with (19), (20) yield the estimate

$$
|y(t)-x(t)| \leqq a\left|y_{0}-x_{0}\right|+\int_{t_{0}}^{t} a \lambda(s)|y(s)| d s+\sum_{t_{0}<t_{k}<t} a \beta_{k}\left|y\left(t_{k}\right)\right| .
$$

Let $c=\sup _{t \in I}|x(t)|$. Then it follows from (23)

$$
|y(t)| \leqq c+a\left|y_{0}-x_{0}\right|+\int_{i_{0}}^{t} a \lambda(s)|y(s)| d s+\sum_{t_{0}<t_{k}<t} a \beta_{k}\left|y\left(t_{k}\right)\right| .
$$


Applying Lemma 1 to (25) one obtains

$$
\begin{aligned}
|y(t)| & \leqq\left(c+a\left|y_{0}-x_{0}\right|\right) \exp \left(\int_{t_{0}}^{t} a \lambda(s) d s\right) \prod_{t_{0}<t_{k}<t}\left(1+a \beta_{k}\right) \\
& =\left(c+a\left|y_{0}-x_{0}\right|\right) \exp \left(\int_{t_{0}}^{t} a \lambda(s) d s+\sum_{t_{0}<t_{k}<t} \ln \left(1+a \beta_{k}\right)\right) \\
& \leqq\left(c+a\left|y_{0}-x_{0}\right|\right) \exp \left(a\left(\int_{t_{0}}^{t} \lambda(s) d s+\sum_{t_{0}<t_{k}<t} \beta_{k}\right)\right) \\
& \leqq\left(c+a\left|y_{0}-x_{0}\right|\right) \exp (a L)<\infty .
\end{aligned}
$$

Therefore, each solution of (2) is bounded and, in view of A3, A5 and A6, is defined on $I$.

If $\bar{y}(t)=y\left(t ; t_{0}, \bar{y}_{0}\right)$ is a solution of $(2)$, then similar arguments yield the estimate

$$
|y(t)-\bar{y}(t)| \leqq M\left|y_{0}-\bar{y}_{0}\right| \quad(M=a \exp (a L)) .
$$

It is easy to see that the following relations hold:

$$
\begin{gathered}
G_{0}(t, s)=G\left(t, t_{0}\right) G_{0}\left(t_{0}, s\right), \text { for } t>t_{0}, s>t_{0}, t \neq t_{k}, s \neq t_{k}, \\
G_{0}\left(t, t_{k}\right)=G\left(t, t_{0}\right)(E+B)^{\omega} G_{0}\left(t_{0}, t_{k}\right), \text { for } t_{0} \in I \text { and } k=1,2, \ldots,
\end{gathered}
$$

where $\omega$ equals $-1,1$ or 0 depending on the mutual deployment of $t_{0}, t_{k}$ and $t$.

It follows from (22) that the matrix

$$
F\left(t_{0}, t_{k}\right)=(E+B)^{\omega} G_{0}\left(t_{0}, t_{k}\right)
$$

can be estimated as

$$
\left\|F\left(t_{0}, t_{k}\right)\right\| \leqq N
$$

where the constant $N>0$ does not depend on $t_{0}$ and $t_{k}$.

Introduce the mapping

$$
y_{0} \mapsto x_{0}, \quad x_{0}=y_{0}+S_{t_{0}}\left(y_{0}\right)
$$

where

$$
S_{t_{0}}\left(y_{0}\right)=\int_{i_{0}}^{\infty} G_{0}\left(t_{0}, s\right) f(s, y(s)) d s+\sum_{t_{0}<t_{k}} F\left(t_{0}, t_{k}\right) b_{k}\left(y\left(t_{k}\right)\right)
$$


Now from the boundedness of $y(t)$, the estimates (23), (30) and conditions (4), (6) and (8) one is able to conclude that for each fixed $t_{0} \in I$ the mapping $S_{t_{0}}\left(y_{0}\right)$ is defined for all $y_{0} \in R^{n}$.

Let $x_{0} \in R^{n}$ be fixed and consider the mapping

$$
U_{t_{0}}: R^{n} \rightarrow R^{n}, \quad U_{t_{0}}\left(y_{0}\right)=y_{0}-S_{t_{0}}\left(y_{0}\right) .
$$

Using conditions (5), (7) and the estimates (23), (30) and (26) we obtain

$$
\left|U_{t_{0}}\left(y_{0}\right)-U_{t_{0}}\left(\bar{y}_{0}\right)\right| \leqq\left(\int_{t_{0}}^{t} a M \lambda(s) d s+N M \sum_{t_{0}<t_{k}} \beta_{k}\right)\left|y_{0}-\bar{y}_{0}\right| .
$$

It follows from (33) and (8) that for $t_{0}$ sufficiently large the mapping $U_{t_{0}}$ is contractive and has a unique fixed point $y_{0} \in R^{n}, U_{t_{0}}\left(y_{0}\right)=y_{0}$, i.e. the mapping (31) is one-to-one. Let us fix such a $t_{0}$.

Since the solutions of (1) and (2) are uniquely determined by the initial conditions $x_{0}$ and $y_{0}$, then the mapping (31) generates a one-to-one correspondence between the solutions $x(t)=x\left(t ; t_{0}, x_{0}\right)$ and $y(t)=y\left(t ; t_{0}, y_{0}\right)$ of these systems.

Now (19), (20), (31), (14), (27)-(29) and (32) yield the following relation between two corresponding solutions

$$
\begin{aligned}
y(t)-x(t)= & \int_{t_{0}}^{t} G_{-}(t, s) f(s, y(s)) d s+\sum_{t_{0}<t_{k}<t} G_{-}\left(t, t_{k}\right) b_{k}\left(y\left(t_{k}\right)\right) \\
& -\int_{t}^{\infty} G_{0}(t, s) f(s, y(s)) d s-\sum_{t \leq t_{k}} G_{0}\left(t, t_{k}\right) b_{k}\left(y\left(t_{k}\right)\right) .
\end{aligned}
$$

Let $K=\sup _{t \in I}|y(t)|$. Then, using (34), (21), (22), (4) and (6) one obtains the estimate

$$
\begin{aligned}
|y(t)-x(t)| \leqq & K a\left(\int_{i_{0}}^{t} \exp (-\alpha(t-s)) \lambda(s) d s+\sum_{t_{0}<t_{k}<t} \exp \left(-\alpha\left(t-t_{k}\right)\right) \beta_{k}\right. \\
& \left.+\int_{t}^{\infty} \lambda(s) d s+\sum_{t \leqq t_{k}} \beta_{k}\right) .
\end{aligned}
$$

It follows from (8) that

$$
\lim _{t \rightarrow \infty}\left(\int_{t}^{\infty} \lambda(s) d s+\sum_{t \leq t_{k}} \beta_{k}\right)=0
$$

Let $t>2 t_{0}$. Then

$$
\begin{aligned}
& \int_{t_{0}}^{t} \exp (-\alpha(t-s)) \lambda(s) d s+\sum_{t_{0}<t_{k}<t} \exp \left(-\alpha\left(t-t_{k}\right)\right) \beta_{k} \\
& \leqq \\
& \quad \exp \left(-\frac{\alpha t}{2}\right)\left(\int_{0}^{\infty} \lambda(s) d s+\sum_{k=1}^{\infty} \beta_{k}\right)+\int_{t / 2}^{\infty} \lambda(s) d s+\sum_{t / 2 \leqq t_{k}} \beta_{k} .
\end{aligned}
$$


With (36) in mind the estimates (35) and (37) yield $\lim _{t \rightarrow \infty}|y(t)-x(t)|=0$.

Thus, Theorem 1 is proved.

Remark 1. In the case when the system (2) is linear $\left(f(t, y)=P(t) y, b_{k}(y)=P_{k} y ; P(t)\right.$ and $P_{k}, k=1,2, \ldots$ are $n \times n$ matrices) Theorem 1 remains valid if A1-A4 hold and A5, A6 are replaced by

A5'. $\quad \operatorname{det}\left(E+B+P_{k}\right) \neq 0, \quad k=1,2, \ldots$.

A6'. The matrix function $P(t)$ is continuous on $I$ and

$$
\int_{0}^{\infty}\|P(t)\| d t+\sum_{k=1}^{\infty}\left\|P_{k}\right\|<\infty
$$

Remark 2. The analysis of the proof of Theorem 1 shows that the Lipschitz conditions (5) and (7) are used only when establishing the uniqueness and continuity of the solutions of (2) as well as the existence of the inverse mapping of (31). These conditions together with (8) restrict the application of Theorem 1 . If one omits the invertibility of the mapping (31), then the following result takes place.

Theorem 2. Let the following conditions be fulfilled:

1. The conditions A1-A5 hold.

2. The functions $f(t, y)$ and $b_{k}(y), k=1,2, \ldots$ are continuous on their domains and there exist a non-negative continuous function $\lambda: I \rightarrow I$ and constants $\beta_{k} \geqq 0, k=1,2, \ldots$, such that

$$
\begin{gathered}
|f(t, y)| \leqq \lambda(t)|y|, \text { for } t \in I \text { and } y \in R^{n}, \\
\left|b_{k}(y)\right| \leqq \beta_{k}|y|, \text { for } y \in R^{n}, \\
\int_{0}^{\infty} \lambda(t) d t+\sum_{k=1}^{\infty} \beta_{k}<\infty .
\end{gathered}
$$

3. The function $f(t, x)$ is locally Lipschitzian in $y$ in the domain $I \times R^{n}$.

Then for each solution $y(t)$ of (2) there exists a solution $x(t)$ of (1) such that $\lim _{t \rightarrow \infty}|y(t)-x(t)|=0$.

\section{REFERENCES}

1. F. Brauer, Nonlinear differential equations with forcing terms. Proc. Amer. Math. Soc. 15 (1964), 758-765.

2. A. M. Samollenko and N. A. Perestuuk, Stability of the solutions of differential equations with impulse effect, Differentsialnye Uravneniya 11 (1977), 1981-1992 (in Russian).

Plovdiv University "P. Hilendarski"

Plovdiv

BULGaria 